La notevole fortuna dell'opera che fu stampala per la prima volta a Perugia nel 1475 e di cui sopravvivono vari manoscritti in biblioteche italiane, e la curiosita del Machiavelli per la commedia elegiaca medioevale cui il De vetula è spesso associata nella tradizione manoscritta,' rendono plausibile una risposta positiva. Se non fosse cosí, dovremmo pensare che la combinatoria della mente umana è alquanto limitata, per cui cra possibile che l'autore del De l'etula c Machiavelli fossero, a distanza di sccoli, capaci di immaginare una situazione analoga che presenta la rara circostanza di trovarsi all insaputa con una vecchia rivoltante a letto in una stanza oscura, circostanza che provoca nel protagonista maschile disgusto fino al vomito una volta che si rende conto d'aver accarezzato o goduto una vecchia laida. ${ }^{2}$

The University of Chicago

\title{
NOTE
}

1 Per il census dei manuscritti e delle edizioni si rimanda all’ampio studio di P. Klopsch alla sua ed. citata.

2 Tanto il Basile quanto il Ferroni indicano una possibile fonte della novellella di Messer Niccolò in una novella del Decameron (VIII, 4). Ci sono, è verò delle similarità. Ma intanto Ciutazza, con cui giace il "prevosto," non c̀ una vecchia; e il disgusto per aver giaciuto con una donna brutta non fa parte del racconto boccaciano.

\section{OPERE CONSULTATE}

BASILE, B. "Grotteschi machiavelliani." Contivium 34 (1966): 576-83.

De vetula. Ed. Paul Klopsch. Leida-Colonia: Brill, 1967.

FERRONI, G. "Le 'cose vane' nelle lettere del Machiavelli," Rassegna della lelleratura italiana, 76 (1972): 215-264.

MARTELLI, M. Una giarda fiorentina. Roma: Salerno Editrice. 1978. 


\title{
Lighea di Giuseppe Tomasi di Lampedusa, ovvero il peso della storia e la leggerezza dell'Essere
}

\author{
Mauro Buccheri
}

"Pur a noi converrà vincer la punga" cominciò el, "se non ... Tal ne s'offerse: oh quanto tarda a me ch'altri qui giunga!"

(Dante, Inf. IX. 7-9)

"E viene un sapiente a curare l'angoscia."

(G. Colli, La sapienza greca, II, 15)

I. In uno dei suoi libri piú recenti, Il tramonto dell'ideologia, Lucio Colletti, descrivendo il clima ideologico degli anni che vanno dal 1968 al presente-ma riferendosi specificamente alla fine degli anni Settanta-scrive: “. . . il tono, lo stimmung di questo particolarissimo momento sembrano interpretati bene ... dalla diffusione ... delle filosofie di Nietzsche e di Heidegger e, in genere, della cultura della cosiddetta disperazione c della crisi” (80). Nella conclusione a cui egli approda, se da un lato rifiuta le proposte per superare il nichilismo provenienti sia da ambienti nietzscheani e heideggeriani che marxisti, ${ }^{1}$ dall'altro deve ammettere di rimanere lucidamente abbarbicato al di qua di una possibile nuova Età della Ragione: "E tuttavia è doveroso riconoscere che i segni di una cultura razionalistica nuova, la cultura dell'età post-idcologica, in Italia non si intravedono ancora" (86).

Se però usciamo dai limiti di pensabilità del marxismo e dello storicismo di Colletti e indietreggiamo nel tempo, sul versante della letteratura, qualche balenare di segni premonitori di crisi e insieme di oltrepassamento della "Krisis" può essere già colto nella plumbea cappa dell'età ideologica e industrialc; e senza ricorrere a mistici salti nell'irrazionale (almeno per ora cosí ci sembra), anzi "immaginando razionalmente," come fa Rosario La Ciura-il "sapiente" 
protagonista di Lighea - un ritorno utopico alle remote origini della civiltà greca, un ritorno che abolisca le dicotomie cristiano-borghesi c marxiste.

Il nostro punto d'esordio è l'areá del Novecento delimitata, da un lato, dalla pubblicazione del Fu Mattia Pascal (1904) e della Coscienza di Zeno (1923), e dall'altro dal meteorico apparire del Gattopardo (1958) e di Lighea (1961). La scelta non c̀ casuale: basta infatti pensare al notevole successo presso il pubblico dei lettori odierni, presi anch'essi dal gorgo della "Krisis," delle citate opere di Pirandello e di Svevo, e ai risultati di un recente sondaggio d'opinione secondo i quali i due romanzi piú letti e piú amati dagli italiani sono Il Gattopardo e La coscienza di Zcno, ${ }^{2}$ due opere chiaramente inscritte nell'area culturale nichilista.

Ora, se Colletti non può ignorare $\mathrm{i}$ pendolari $\mathrm{e}$ vistosi ritorni dell'"irrazionale" nel pensicro e nella prassi politica di questo nostro tardo Novecento (né puo fare a meno di augurarsi un ritorno alla razionalità), noi non possiamo trascurarne la presenza nella letteratura, ma-come vedremo-per ben altre ragioni $\mathrm{e}$ in vista di altri itinerari e mete. Va subito detto che la letteratura è qui considerata un campo di indagine molto piú disponibile a ristrutturazioni-diciamo pure a operazioni di "restauro" anche spregiudicate—e piú aperto quindi a interpretazioni radicali di quanto non lo siano le scienze umane e sociali (e forse anche la stessa filosofia). La componente "fantastica" è in letteratura, per definizione, dominante; e i progetti utopici sono piú "a portata di mano" perché non sottoposti o sottoponibili a immediata verifica sul reale (e quindi alla tirannia dei fatti), ma non per ciò meno pregnanti e suscettibili di future attuazioni. In fondo l'impasse di cui parla Colletti è un'impasse dell'immaginazione, un cedere troppo alle intimazioni delle "situazioni oggettive," una svalutazione delle potenzialità del soggetto, tutto sommato anch'essa "nichilista" pur se proveniente dalle spiagge del razionalismo e dello storicismo marxista. A ciò si aggiunga che la crisi c la dissoluzione del soggetto cristiano-borghese talvolta sono fatte coincidere troppo frettolosamente con la dissoluzione del soggetto tout court, come se non fosse affatto possibile immaginare un "al di là" del soggetto attuale. E a questo "al di là" che il nostro sguardo vuole volgersi: non per cercare i segni premonitori d'un'età razionalista, ma piuttosto la liberazione dell'immaginazione e dell'Eros imbrigliati da categoric 
"violente" e metafisiche di cui la razionalità borghese e anche quella marxista non sono che manifestazioni alienate e alienanti (Severino, Gli abitatori 9-115).

II. Se il Pensicro dialettico è in piena crisi dunque, e ritorna in questo tormentato fine secolo, come nell'altro, il Pensiero negativo, da quale "soggetto" dovrebbe partire l'iniziativa di inventare la nuova età? Quali il centro e gli orizzonti, i tempi e i luoghi, gli umanesimi e gli illuminismi della vita nuova? Nessuno lo sa: si sono eclissati i profeti e le sibille. Eppure nell' "officina" del poeta qualche segno balugina ambiguo in attesa d'essere interpretato. Il "sapiente"-per usare una parola cara a Colli, ma che appare piú d'una volta in Lighea ${ }^{3}$-deve saper sciogliere enigmi o perire poiché al centro del "mondo" non c'è la trasparenza assoluta del "logos" ma l'obliqua opacità del "mythos." Interroghiamo allora un sapiente moderno: questa volta non Svevo o Pirandello, ma Lampedusa, a noi piú vicino nel tempo, caso letterario singolare fin dall'inizio e, tuttora, autore emarginato dalla critica. Interroghiamo Lampedusa per tentare di sciogliere non l'enigma del Gattopardo ma quello di Lighea. Si tratta, come vedremo, di lanciare una sfida alla concezione della storia cristiana, borghese e marxista, ma ciò è possibile solo se l'intellettuale si sente di nuovo in grado di sciogliere enigmi. E se la ragione lo precipita nel "mare dell'oggettività," nel labirinto del "Cosí è," l'immaginazione accetti la sfida e sia la "favola saggia" l'ala che lo sollevi fuori del labirinto se non erra Nietzsche quando scrive: "Ma l'uccello saggezza parla cosí: Ecco, non c'è sopra né sotto! Slanciati c vola: in giro, in avanti, all'indietro, tu che sei lieve!" (Nietzsche 282). E quale testo di questo nostro greve Novecento è piú "leggero" di Lighea? Quale dialettica piú dionisiaca e vitalismo piú apollineo? Dove si indica ai mali dell'uomo occidentale un rimedio piú certo e insieme piú problematico? Tuttavia ciò diventa palese solo se si legge Lighea in filigrana, con il pensiero di Nietzsche nell'interpretazione di Colli e di Vattimo e con la filosofia di Emanuele Severino presente all'orizzonte interpretativo (Colli, Dopo Nietzsche, Scritti su Nietzsche; Vattimo, La fine; Severino, Gli abitatori, L'essenza): soltanto cosí la "decadente" Weltanschauung borghese e l'impasse marxista possono essere superate, e gli Agosti Incsauribili recuperati dal limbo intrastorico in cui sono precipitati 
dopo i secoli della sapienza grecal.

Se la tragedia dell'uomo occidentale scaturise dalla visione del mondo divisa del cristianesimo e della borghesia, l'ingorgo attuale è nel riconoscere, ed accettare, senza illusione alcuna ormai, che la separatezza c̀ abissale e tuttora insuperata. Ligheoa è la risposta della fantasia poetica di Lampedusa a tale ingorgo: la nostra lettura del racconto infatti vuole proporre una concezione del mondo dello scrittore siciliano non piú rigidamente chiusa nella struttura concettuale nichilista ma in movimento dal nichilismo del Gattopardo all'inevitabile "entropia" borghese dei Gattini ciechi c infine, procedendo ancora oltre, in movimento verso i lembi estremi dove la storia si rovescia in mito; un mito il cui statuto utopico non significa però irrealtà o surrealtà ma progetto "in avanti," "Ontologia del non ancora" (Bloch, Marx, Experimentum, The Principle), o paradossalnente "del non piú" che deve essere fatto riaffiorare dal fondo della storia. Ma, sia ben chiaro, l'utopia rimane racchiusa in una ferrea cornice storica; l'oltrepassamento è solitaria conquista dell'illustre filologo; la coincidenza di Apollo c Dionisio, di divinità c animalità, di Eros e Tanatos (Lampedusa 86-89) sono possibili solo per chi scelga di abitare fuori del tempo; nel tempo tornano le stagioni-Gli Agosti non sono piú Inesauribili e si spengono nelle Feste della bufera ${ }^{5}-\mathrm{e}$ Tanatos è principio distruttore che con pari forza si oppone a Eros (non piú come nell'eternità dell'Essere la sua pallida ombra). Alla fine del racconto, sfiorata appena l'essenza dell'enigma, il percorso storico rimane inconcluso e votato al nulla: simbolo di questa storia monca sono i picdi di Ulisse effigiati in un frammento di cratere greco ritrovato a Palermo, fra i ruderi del palazzo dei Corbera, distrutto dalle bombe dei "Liberators" americani."

La Weltanschauming di Lampedusa da un lato punta ancora sull'approfondimento della tematica dell'ascesa al potere dei borghesi, dall' altro cerca dentro la malattia storica, nella millenaria civiltà greca (i cui millenni si oppongono ai secoli delle altre civiltà), il germe salvifico. In effetti Lighea introduce nella concezione del mondo lampedusiana un elemento "nuovo," dal potenziale concettuale "rivoluzionario" se inserito nel quadro culturologico a cui ci siamo richiamati con i riferimenti a Colletti, Nietzsche, Vattimo e Severino. L'operazione dello scrittore siciliano ci sembra una delle piú radicali fra quelle tentate, sul versante dell utopia, nel nostro Novecento. ${ }^{7}$ Dicia- 
mo subito che di primo acchito può persino sorprendere l'affermazione stessa di una presenza utopica, per quanto problematica, nella visione del mondo lampedusiana. Tuttavia chiudendo saldamente il racconto fra rimemorazione proustiana e "oblio" nietzscheano, fra il peso della storia-memoria e la "leggerezza" dell'essere-oblio, rimemorando il lontanissimo passato dell'umanità attraverso il disseppellimento dall'intrastoria degli Agosti Inesauribili e obliando tutta la storia cristiano-borghese e gran parte anche di quella greca, Lampedusa propone proprio un utopico ritorno al presocratico, anzi all'età preomerica, che ci sembra simile a quello proposto da Severino in Gli abitatori del tempo e in L'essenza del nichilismo. ${ }^{8}$ Il professor Rosario La Ciura, illustre grecista, rinuncia a tutte le contingenze del mondo storico borghese perché è stato abitatore degli Agosti Inesauribili: e quando, ormai vecchio, torna a Lighea, egli non è vinto dalla cupio dissolvi, come ha creduto la critica: in realtà ha deciso di abbandonare il fragile mondo della storia per tornare all'eternità dell'Essere, alla Gioia immortale dell'Eros.'

A questa operazione tematico-concettuale si affianca un'operazione estetica di alto livello, ma non è qui la novità di Lighea. La sua attualità è nell'insolita e prefigurante utopia se il nostro accostamento a Severino regge. ${ }^{10}$

III. Fin dalla prima metà del Novecento, dopo aver abitato a lungo il versante della pars destruens, Luigi Pirandello, Italo Svevo, Elio Vittorini e Cesare Pavese incominciano a progettare le prime utopie. Se l'utopia non si configura come progetto positivo, domina certamente come progetto "negativo." "Negli anni che vanno dal 1965 al 1975, l'utopia come progetto fondante o la speranza utopica dominano non solo la cultura italiana, ma anche quella curopea ed americana. L'essenza del nichilismo (la prima parte è appunto intitolata "Tornare a Parmenide") appare nel 1972. Se questo, qui sommariamente descritto, è il paesaggio culturale in cui collocare Lighea, facciamo ora emergere da esso uno scrittore particolarmente congeniale a un raffronto con Lampedusa. Facciamo un rapido raffronto con le utopie proposte da Pavese in anni che precedono di poco la pubblicazione del Gattopardo e dei Racconti, senza dimenticare d'altro canto il contesto culturale attuale. L'utopia in Pavese finisce col configurarsi come utopia negativa: Paesi tuoi, Il compa- 
gno, Dialoghi con Léucò, La luna e i falò (Tutto Pavese). Se letti dat questa angolatura lo dimostrano agevolmente. Prendiamo per esempio Il compagno: l'utopia qui è quella marxista del ritorno al popolo dell'intellettuale piccolo-borghese; è ben noto il fallimento di questo "andare verso il popolo" sia di Pavese che degli intellettuali marxisti. Paesi tuoi è il libro del ritorno del represso: l'esplosione improvvisal del dionisiaco che si configura come dolore e terribilità del vivere; tornare alla civiltà contadina, al preindustriale, rivela la radice incestuosa della sessualità umana, comporta violenza $\mathrm{c}$ morte. La luna $e$ i falò è la sintesi conclusiva della pavesiana utopia negativa: al tema circolare ed elegiaco del grande ritorno alle origini si contrappone la tragica e brutale linearità della storia: nelle pagine finali il mondo contadino e quello storico si saldano nel simbolo di morte del letto del falò. Quello che doveva essere un gioioso viaggio alle origini si rivela regressione a tanatos, tragicità del vivere, dentro o fuori della storia. I dialoghi con Leucò, infine, sono una interpretazione in chiave esistenzialistica del mondo greco. Vi domina la brutalità dionisiaca non temprata da un momento apollineo che rassereni $\mathrm{e}$ purifichi; oppure si insinua una dolorosa vena elegiaca. Il ritorno ai Greci è ritorno senza utopia, un rivivere il mondo antico in chiave nichilista.

Dei testi sin qui rapidamente descritti, I dialoghi con Leucò è quello che permette un confronto piú proficuo con Lighea. I dialoghi furono scritti fra il 1945 e il 1947; Lampedusa scrive Lighea nel 1957: li separa quindi circa un decennio; entrambi costituiscono un simbolico ritorno allc origini greche arcaiche; in entrambi domina la figura di una divinità greca che sembra costituirc il nucleo enigmatico della visione del mondo di Pavese e di Lampedusa; inoltre: il primo titolo di Dialoghi con Leucò cra Uomini e dei (Pavese 175) c il professor La Ciura ha scritto un libro intitolato Uomini e dei (Lampedusa 59). Leucò è la dea Leucotca, la divinità che soccorre Ulisse naufrago su una zattera in balia alle onde: ella porta una tunica che gli salverà la vita se lo vorrà. ${ }^{2}$ Ulisse, archetipo dell'uomo occidentale borghese (Andreac "Ulisse," L'immagine 3-8), che vuole essere padrone del proprio destino, accetta l'aiuto ma userà la veste solo se non riuscirà a mettersi in salvo con le propric forze. Il che se sottolinea l'orgoglio ulissiaco di voler fare a meno degli dei (nasce cosí l'archetipo dell'uomo occidentale diventato solitario abitatore 
del tempo), rivela oggi la profonda nostalgia delle origini, la perdita della certezza dell'Essere.

Non usando la tunica Ulisse avvia il processo di allontanamento dell'Essere. Pavese cosí vede nel mondo greco il progetto fondante la terribilità della vita, l'origine della tremenda solitudine dell'uomo moderno, incapace ormai di pensare al mito come metafora del reale, all'utopia come progetto del "non ancora," completamente padrone del proprio destino grazie agli "Ordigni" come ironicamente direbbe Zeno: ${ }^{13}$ il destino di una umanità ulissiaca quindi c̀ un destino di solitudine, di inguaribile inquietudine e di morte.

L'alternativa è Lighea: la sirena che tentò di far tornare all'Essere il tracotante Ulisse. Sc Ulisse rifiuta il richiamo delle sirene, La Ciura si lascia incantare. Il mondo greco, per questo Oltreuomo siciliano, è il mondo in cui il dolore si annulla nella Gioia, Dioniso confluisce in Apollo; in cui dialettica e divinazione s'innestano l'una sull'altra (Colli, La nascita 13-21, 39-46, 73-81) c la morte diventa ombra labile della vita, mero riflesso temporale dell'Essere. Ulisse rimane mortale abitatore del tempo: l'umanità tutta vive come dei "gattini ciechi," tesa soltanto ad accumulare "robba" e ad accelcrare per mezzo della tecnologia la propria corsa verso l'autodistruzione. Perché, contrariamente alla tradizione che lo esalta-da Omero a Dante a Tennyson, allo stesso Joyce-l'archetipo ulissiaco viene bruscamente degradato e fatto tramontare da Lampedusa? Perché, come dimostra Giorgio Colli in La nascita della filosofia (15), solo il "sapiente" accede all'enigma e sa scioglierlo: Ulisse non è un "sapiente," è un proto-borghese tutto preso dalle conquiste "terrene," tutto teso al dominio sugli altri, alla sopravvivenza ad ogni costo, pronto a ricorrere alla frode pur di ottenere i propri scopi; Ulisse vive nella "via dell'apparenza" e il sapiente nella "via della verità"; c non è possibile riconciliare "Alétheia" e "Doxa," verità e retorica. Rosario La Ciura, per contro, è il sápiente (a ciò allude anche il vezzeggiativo Sasà [87]) che ha vissuto dentro l'enigma, conosce la via dell'Essere e si solleva, ma senza fare violenza agli altri, al di sopra della fragilità della storia cristiano-borghese. L'ulissismo pavesiano è nichilista; la figura di Ulisse, anche in Lighea, è simbolo di distruzione, di cupio dissolvi; ma se per Pavese il tramonto del mito di Ulisse comporta il Nulla, la tragicità-tutta nietzscheana-di una storia senza "valori" e senza "senso" che in se stessa s'ingorga 
e si spegne, per Lampedusa tale tramonto è necessario affinché un altro mito si sostituisca ad esso: il mito della coincidenza di vita c conoscenza nel ritorno a Lighea.

IV. Lampedusa scrisse Lighea nell'estate del 1957, poco prima della morte. Secondo quanto afferma il figlio adottivo, Francesco Orlando in Ricordo di Lampedusa, lo scrittore siciliano amava di un amore particolare quest'ultima opera (Salvestroni 110). Da Giuseppe Paolo Samonà sappiamo, inoltre, che c'è anche un'edizione di Lighea registrata dallo stesso Lampedusa: “. . importante non solo . . . come documento diretto biografico sia pure molto accessorio, ma anche e soprattutto in quanto ci rende-attraverso i vari toni $\mathrm{e}$ l'atteggiarsi della voce - parte non indifferente dell'animus lampedusiano verso questa sua opera, e della interpretazione che egli stesso ne dà ..." (Samonà 327, nota 21 ). A parte ciò che giustamente dice Samonà, il fatto stesso che ci sia un'edizione registrata dall'autore rafforza il valore della testimonianza di Orlando sulla predilezione di Lampedusa per quesıo racconto. Abbiamo in mano, cosí, due elementi da non trascurare nello sforzo interpretativo, se non proprio sul piano critico (in senso stretto), perlomeno su quello psicologico; a Lighea è affidato l'ultimo messaggio di Lampedusa, ma perché è il testo prediletto? Come si è già accennato crediamo che la ragione piú intima risieda nella forma mitica del contenuto che, se vagliata attentamente, rivela una visione del mondo in movimento verso posizioni radicali e sorprendentemente moderne, di cui l'autore doveva pur essere almeno in parte consapevole. E, infine, un'edizione critica di Lighea, condotta sia su quella registrata che sul manoscritto, sarebbe molto utile (si veda a questo proposito Samonà) e ai fini di una interpretazione globale di Lampedusa e a quelli specifici di una riattivazione del testo in chiave utopica per inserirlo nel dibattito culturale attuale.

È certo, comunque, che Lighea, come del resto l'intera raccolta dei Racconti, non ha ricevuto l'attenzione che merita: non solo su un piano squisitamente letterario, ma anche su quello delle strutture concettuali c dei matcriali antropologici; una lettura, anche sommaria, della critica (stranamente sparuta) lo conferma agevolmente. ${ }^{14}$

Occorre allora cominciare il viaggio nel testo dalle tecniche d'esposizione per poi scendere nel fondo della sua legge generativa, il sot- 
tosuolo concettuale. La struttura del modello narrativo è divisa c cosí pure la voce poetica. La superficie espositiva riflette la sottostante dicotomia concettuale: da un lato c'è la cornice storica, introdotta in prima persona da Paolo Corbera, il giovane siciliano di nobile famiglia che lavora per un giornale di Torino; egli rappresenta il punto di vista "mortale," e occupa da solo il prologo e l'epilogo del racconto in modo da essere il filo conduttore della storia concepita come fallimento di Ulisse archetipo del superuomo borghese (e come fallimento del cristianesimo e del fascismo); dall'altro l' "avventura," narrata sottovoce, in un'atmosfera essoterica, di prodigio imminente, dal professor La Ciura, ${ }^{15}$ punto di vista immortale, o metastorico. La forma concettuale del "prodigio" è quella circolare del mito del ritorno, dell'Eros eterno (della sintesi di sápienza e animalità, di gioia sensuale e conoscenza intellettuale), mentre nella cornice imperversa la linearità di Tanatos. Questi due nuclei concettuali si sorreggono a vicenda ma si integrano solo sul piano estetico. All'altezza degli altri tagli si tratta di pura contiguità, della necessità di riflettere la concreta situazione umana: la dicotomia fra Essere e tempo che la caratterizza.

Se il lettore compie il viaggio testuale insieme a Paolo Corbera, passerà dall'iniziale scetticismo alla certezza, emergente nelle ultime pagine, che il vecchio professore ha detto lá verità: ma cosí sí ritrovcrà gettato nella sconsolante storia ulissiaca dell'epilogo, escluso dall'utopia degli Agosti Inesauribili; se, per contro, l'esperienza della lettura è compiuta secondo il "punto di vista" di La Ciura, egli si troverà sí diviso fra storia e utopia, ma con la certezza del ritorno a Lighea (attuabile in qualsiasi momento). C'c̀, tuttavia, un altro "punto di vista," che pensiamo costituisca il sottosuolo a cui rinvia il testo nella ricerca della sua leggc piú profonda: ed è quello di Lighea.

Se entriamo "dentro" la sirena, ciò che ai critici (perché tutti presi dal Gattopardo) è sembrata una "favola" surreale, una fuga dalla realtà (poiché essi hanno voluto farc l'esperienza estetica sociologicamente, da "fuori," e non fenomenologicamente, da "dentro"), a noi apparirà come realtà mitica da far emergere dai fondali inesplorati dell'intrastoria. Solo leggendo Lighea dal sottosuolo mitico, l'utopia può essere ribaltata in realtà. L'esperienza estetica deve condurre il lettore, se non proprio dentro l'enigma, almeno nella sua massima 
prossimità: e questa può essere raggiunta solo calandosi in Lighea.

Tuttavia, il racconto, se richiede questa "disecsa" dalla "superficic" storica (e stilistico-narrativa) al sottosuolo concettuale, nelle ultime pagine riconduce alla "superficie," alla storia che si congeda da noi, per ora, con l'immagine dei bombardamenti americani e di Ulisse mutilato. Conclusasi la lettura di Lighea, sccondo le modalità qui schematicamente descritte, occorre- è chiaro-fare un bilancio sul piano della storia letteraria e su quello concettualc $\mathrm{c}$ storico (come Robinson Crusoe, dopo il naufragio e l'immersione in profondità, bisogna tornare sulla spiaggia e cominciare a ricostruire la vita quotidiana). Si tratta di vedere se "usciti" dal testo, l'utopia regge al confronto con "la realtà," se ciò che nel testo sembrava piú prezioso, lo è ancora alla luce del contesto esistenziale e storico del "qui e ora"; bisogna, insomma, fare i conti con "l'ombrosa ragionc," come direbbe La Ciura, - non solo nostra ma anche degli altri-che recalcitra dinanzi al prodigio. ${ }^{16}$

V. Il nostro percorso è giunto ora ad un altro bivio, quasi come nel borgesiano "jardin de senderos que se bifurcan" (111-116): di qua il "salto" nel misticismo; di là quanto rimane di "impensabile" nell'ambito del Pensiero negativo. L'ombrosa ragione ci impedisce di andare oltre. Eppure all'utopia come "ontologia del non ancora" o come "vento della storia" non sappiamo né vogliamo rinunciare.

Tornare a Lighea non significa rifiutare la storia tout court, ma la storia borghese, la "violenta" tradizione ulissiaca: c Lighea è l'Essere appena intravisto all'alba della sapienza greca, quando non erano cominciate le avventure della "differenza." Qui, in limine, accanto all'enigma ci fermiamo, come Heidegger dopo il poderoso sforzo di interpretare il mito dell'eterno ritorno e la dottrina dell'oltreuomo: ". . this most abysmal thought conceals something unthought. something which at the same time remains a sealed door to metaphisical thinking" (Heidegger 233). Se il volto "vero" di Zarathustra è destinato, almeno in parte, a rimanere un enigma la cui cssenza piú misteriosa si esprime nei simboli dell'aquila e del serpente. l'utopia di Lighea sfugge alla ragione nella misura in cui si dimentica che lo "scioglitore" di enigmi si esprime anch'egli per enigmi: come si conviene al sapiente, cosí parla obliquamente, per simboli, anche Rosario La Ciura, incarnazione lampedusiana dell'oltreuomo 


\section{nietzscheano, o Zarathustra di Sicilia:}

Questo qui-aggiunse prendendo in mano il mio Shakespeare-questo qui qualche cosa la capiva.

A sea change into something rich and strange. What potions have I drunk of syren tears? (Lampedusa 74)

\section{York University}

\section{NOTE}

1 Si veda a questo proposito Vattimo, Il soggetto e Le avventure; Cacciari, KRISIS e Pensiero.

2 Cf. Tuttolibri, 438, 439, 440, 441, 442 e 443 (in quest'ultimo numero si pubblicano i risultati del referendum: "L'autore del secolo è Svevo, il romanzo piú amato /l gattopardo").

3 Cf. Colli, La sapienza 1, 15-48; Lampedusa 59, 89 e 91; (ma la nozione di sapienza-saggezza percorre "tutto" il racconto, ne costituisce l'essenza).

4 Qui ci riferiamo alla dimensione naturalistica del "Cosí è" aspramente erosa da L. Pirandello e al titolo di un articolo di I. Calvino, "ll mare dell'oggettività" apparso per la prima volta in "ll menabò di letteratura" (1960) ed ora ristampato in Calvino 39-45.

5 Cosí si congeda da Rosario La Ciura Lighea: "Voglio restare ancora con te; se adesso andassi al largo i miei compagni del mare mi tratterrebbero. Li senti? Mi chiamano ... Suonano le loro conche, chiamano Lighea per le feste della bufera . . Adduio Sasà. Non dimenticherai." Lampedusa 92.

6 Questa c̀ la conclusione di Lighea: "Poi venne la guerra e mentre io me ne stavo in Marmarica con mezzo litro di acqua al giorno i 'Liberators' distrussero la mia casa: quando ritornai la fotografia era stata tagliata a striscioline che crano servite come torce ai saccheggiatori notturni; il cratere era stato fatto a pezzi; nel frammento piú grosso si vedono i piedi di Ulisse legato all'albero della nave. Lo conservo ancora. 1 libri furono depositati nel sottosuolo dell'Università ma poiché mancano i fondi per le scáffalature essi vanno imputridendo lentamente." Lampedusa 93-94.

7 Si pensi all'ultimo Svevo (in particolare alla sua nozione di "abbozzo dell'uomo del futuro"), a I Miti di Pirandello, al Vittorini de Le donne di Messina e Le città del mondo, al Pavese de $/ 1$ compagno.

8 Naturalmente non si deve dimenticare la diversità dei contesti culturali $\mathrm{e}$ la provenienza da campi di "ricerca" certo non omogenei, come sono la letteratura e la filosofia, di Lampedusa e Severino. Qui a noi interessa soprattutto utilizzare i "nuovi" orizzonti culturali per riattivare l'utopia lampedusiana, per rimetterla in movimento e farla uscire dal limbo culturale in cui è caduta. 
9 "ll giorno dopo, all'alba, si telefonò da Genova al giornale: duranle la notte il senatore La Ciura era calduto in mare dalla coperta del Rex che navigava verso Napoli, e benche delle scialuppe fossero state immediatanente messe in mare, il corpo non era stito ritrovato." Lampedusa 97.

10 "L’utopia" di Severino (giudicata negativamente e ambigua da Vattimo, La fince 13) c̀ quella di "tornare a Parmenide." A noi sembra, pur trovando lo scritto di Severino arduo e spesso enigmatico, che le "intenzioni" del filosofo siano "trasparenti"; e sc illuminiamo "Tornare a Parmenide" con un fascio di luce proveniente da Lighea, non pochi dubbi sono dissipati. Forse è piú facile accettare l'utopia in letteratura che non in filosofia; ma la questione ormai c̀ un'altra: si tratta di riuscire a riattivare l'immaginazione c di uscire dal torpore del "Cosí è," di non lasciarsi sopraffare dal "mare dell'oggettività"; e, se Ietteratura e filosofia convergono e s'incontrano sul terreno dell'utopia, tanto di guadagnato.

11 In questo quadro si possono collocare vari scrittori del secondo Novecento, da Calvino a Volponi, da Pasolini a Sanguineti, a Morsclli.

$12 \mathrm{Cf}$. Andreac, "Ulisse," L 'immagine 3-8.

13 Si leggano le ultime pagine de La coscienza di Zeno per una ironica profezia del tragico destino dell'uomo tecnologico.

14 A parte l'ottima monografia di Samonà (che contiene anche un ampio capitolo su Lampedusa e la critica) e l'esile volumetto della Salvestroni (la quale scrive a pag. 121: "Negli ultimi anni pochissimi critici si sono occupati di Tomasi Di Lampedusa") da quindici anni a questa parte poco è stato scritto su Lampedusa.

15 "Per spiegarmi, però, dovrò raccontarti la mia avventura che è inconsueta . . Si mosse dal proprio posto dietro la scrivania, venne a sedersi sul mio stesso divano. 'Scusa, sai, ma dovrò parlare a bassa voce. Lc parole importanti non possono essere berciate'": Lampedusa 79.

16 "La nostra ombrosa ragione, per quanto predisposta, s'inalbera dinanzi al prodigio e quando ne avverte uno cerca di appoggiarsi al ricordo di fenomeni banali"; Lampedusa 84.

\section{OPERE CONSULTATE}

Andreae, B. L'immagine di Ulisse. Torino: Einaudi, 1983.

Bloch, Ernest. Marx. Bologna: il Mulino, 1972.

Religione in eredità. Brescia: Queriniana, 1979.

Experimentum mundi. Brescia: Queriniana. 1980.

The Principle of Hope. Cambridge: MIT press, 1986.

Borges, J.L. Ficciones. Madrid: Alianza editorial, 1971.

Cacciari, Massimo. Krisis. Milano: Feltrinelli, 1976.

Pensiero negativo e razionalizazione. Venezia: Marsilio, 1977.

Calvino, Italo. Una pietra sopra. Torino: Einaudi, 1980.

Colli, Giorgio. La sapienza greca. Milano: Adelphi, 1981.

Dopo Nietzsche. Milano: Adelphi, 1974. 
Scritti su Nietzsche. Milano: Adelphi, 1980.

La nascita della filosofia. Milano: Adelphi, 1981.

Heidegger, M. Nietzsche: the Eternal Recurrence of the Same. San Francisco: Harper and Row, 1984. Vol. Il.

Horkheimer, M.-Adorno, T.W. Dialettica dell'illuminismo. Torino: Einaudi, 1966.

Nietzsche, Friedrich. Cosí parlò Zarathustra. Vol. VI, Tomo I, "Opere di Friedrich Nietzsche." Milano: Adelphi, 1979.

Pavese, Cesare. Tutto Pavese. Torino: Einaudi, 1968.

Salvestroni, S. Tomasi di Lampedusa. Firenze: La Nuova Italia, 1979.

Samonà, G.P. Il Gattopardo, i racconti, Lampedusa. Firenze: La Nuova Italia, 1974.

Severino, Emanuele. Gli abitatori del tempo. Roma: Armando, 1978. - L'essenza del nichilismo. Milano: Adelphi, 1972.

Svevo, Italo. La coscienza di Zeno. Pordenone: Studio Tesi, 1985.

Tomasi di Lampedusa, Giuseppe. Racconti. Milano: Feltrinelli, 1961.

Vattimo, Gianni. Il soggetto e la maschera. Milano: Bompiani, 1974. . Le avventure della differenza. Milano: Garzanti, 1980. . La fine della modernità. Milano: Garzanti, 1985. 